\title{
CROWDSOURCING PARTICIPATIVO INSTITUCIO- NAL. INFORMACIÓN GEOGRÁFICA VOLUNTARIA EN LA ADMINISTRACIÓN PÚBLICA. EJEMPLOS
}

\author{
Jordi GUIMET PEREÑA \\ Infraestructura de Datos Espaciales de Cataluña - IDEC. Instituto Cartográ- \\ fico y Geológico de Cataluña
}

Recibido: 25/11/2014

Aceptado: $30 / 07 / 2015$

RESUMEN: Se defiende y argumenta que las entidades públicas, tales como administraciones locales, organismos y empresas públicas, fundaciones, etc., están realizando determinadas actividades equivalentes a la VGI, a través de las cuales reúnen y comparten nuevos datos e información geográfica entre ellos y con la comunidad. Y lo hacen, mayoritariamente, por la intervención e iniciativa de algunos de sus miembros, que desde su rol de funcionarios de dichas entidades participan en el mismo esfuerzo de cooperación y contribuyen con la creación, gestión y publicación de información que, en rigor, no pertenece a las tareas asignadas o responsabilidades de dichas entidades públicas.

El artículo presenta algunas experiencias en el marco de la IDE (Infraestructura de Datos Espaciales) catalana que demuestran claramente la importancia creciente de este tipo de voluntariado «público» dentro del paradigma VGI y sus beneficios para las administraciones públicas y los ciudadanos

$P A L A B R A S C L A V E$ : VGI, Voluntariado en Información Geográfica, Infraestructuras de Datos Espaciales, Neogeografia, crowdsourcing, organizaciones públicas, IDEC.

PARTICIPATORY INSTITUTIONAL CROWDSOURCING. EXAMPLES OF INFORMATION GEOGRAPHIC VOLUNTEER IN PUBLIC ADMINISTRATION

ABSTRACT: Public bodies such as local authorities, public organizations and businesses, foundations, etc., are conducting certain activities equivalent to Volunteered Geographic Information (VGI), through which they gather and share new data and geographic information among themselves and with the community. And they do it, mostly, by the intervention and initiative of some of its members who are involved in the same effort of cooperation and contribute to the creation, management and publication of information that, strictly speaking, does not belong to the assignments or responsibilities of such public entities.

The paper presents some experiences in the context of the Catalan SDI (Spatial Data Infrastructure) that clearly demonstrate the growing importance of this type of «public» volunteering within the paradigm VGI and its benefits for public administrations and citizens.

KEY WORDS: VGI, Volunteered Geographic Information, Spatial Data Infrastructures, Neogeography, crowdsourcing, public organizations, IDEC. 


\section{INTRODUCCIÓN}

Quizás la más extendida y conocida actividad dentro del entorno que conocemos como Neogeografia es el Voluntariado en Información Geográfica (siglas VGI en inglés, un término acuñado por M. Goodchild). A su vez dicho fenómeno se engloba en el nuevo fenómeno social de la participación y compartición voluntaria de los ciudadanos en diversos quehaceres, desde la financiación de proyectos (crowdfunding), a la compartición de bienes (collaborative consumption).

El fenómeno de la IG Voluntaria (VGI) o «Geo-crowdsourcing» usualmente se refiere a la recopilación, intercambio y publicación de la información geográfica por voluntarios. Con unas particulares características frente a la clásica producción por organismos oficiales, entre ellas: datos no estructurados frente a modelos definidos, datos actuales (incluso en tiempo real) frente a datos/instantáneas históricos, distribución ubicua y libre frente a licencias y derechos de propiedad, datos no validados frente a calidad de datos garantizada....

El rápido crecimiento de VGI en los últimos años es un paso más en un largo proceso que comenzó hace casi dos décadas, y es probable que continúe durante algún tiempo por venir. Es una parte de una transición fundamental, de cómo la sociedad percibe el papel de la información pública en los primeros años del siglo XXI (GOODCHILD, 2008).

En el contexto del documento, el término «usuario» se refiere a cualquier persona que utiliza una variedad de herramientas para la obtención de un resultado. En el área de la Geoinformación, el usuario es aquella persona que busca, por ejemplo, una dirección o localización, o bien visualizar información o mapas en un contexto geográfico determinado, la mejor ruta, o cualquier otro servicio de Internet relacionado con el territorio y la localización. Pero este usuario también puede recopilar, introducir y compartir información con la comunidad, contribuyendo así, como «voluntario», a la promoción del paradigma de la IG voluntaria.

$\mathrm{Y}$, con las herramientas actuales, la experiencia y conocimientos de dicho usuario ha dejado de ser una base para poder juzgar o atribuir una credibilidad determinada a la información por él producida, aunque es cierto que otros problemas van parejos a la consideración y utilidad de dicha información.

\section{EL ESTADO DE LA SITUACIÓN. ALGUNOS EJEMPLOS}

Afirmamos que el paradigma VIG incluye también aquellas iniciativas de los organismos públicos que contribuyen con la generación y publicación de 
información cuya gestión no deriva estrictamente de sus deberes o responsabilidades públicas. Evidentemente, dado su carácter voluntario, dicha actividad no forma parte de sus procesos de gestión corporativa, sino que se inicia a partir de la aceptación de las sugerencias de la dirección corporativa o de la propia iniciativa de los funcionarios de dichas entidades, que asumen ciertas responsabilidades voluntariamente.

Aunque en términos de números absolutos la importancia de estas organizaciones e individuos podría ser insignificante en comparación con los millones de usuarios que interactúan con Globos Virtuales para un sinfín de aplicaciones, se puede afirmar que la validez, la calidad y la seguridad de la información es mayor que la ordinaria VGI, y de gran interés para la mayoría de los ciudadanos.

El fenómeno VGI de organismos públicos (que denominamos crowdsourcing institucional) tiene varios ejemplos en el marco de la IDEC, la Infraestructura de Datos Espaciales de Cataluña y, más concretamente, dentro de la red IDEC.Local que conforman más de 450 municipios que utilizan los recursos de la IDEC (datos y aplicaciones) para la creación y personalización de aplicaciones diferentes.

Desde el año 2008, 152 municipios han publicado 4.500 capas que contienen una variedad de información referenciada al territorio, tales como rutas turísticas, ubicación de centros cívicos, puntos de interés, instalaciones deportivas y equipamientos generales, itinerarios turísticos que ofrece el sistema de transporte urbano, sitios de patrimonio histórico, de ocio y gastronomía, etc. Una parte importante de esta información no deriva de ninguna responsabilidad, compromiso u obligación formal, sino, fundamentalmente, a partir de la iniciativa espontánea de los funcionarios activos y entusiastas que producen información muy diversa de interés general que complementa la información básica y administrativa que gestionan y a la que tienen acceso.

No se trata de los datos «oficiales», aquellos que se crean y gestionan en virtud de los procesos administrativos y jurídicos que son propios de la organización pública, sino de nuevos datos o, mejor aún, de datos convertidos en objetos espaciales georeferenciados y mapificados, que se encuentran en la esfera de conocimiento del organismo aunque no sean objeto de su gestión (a título de ejemplo: rutas turísticas, recorridos de transportes públicos, localización de enclavamientos turísticos, etc.). También se extiende esta voluntariedad a la mejora de la calidad de los datos existentes, que, aportados por otras instituciones, pueden ser mejorados y validados por otras, en colaboración a través de proyectos participativos basados en sistemas distribuidos (por ejemplo datos de la Comunidad Autónoma que pueden ser mejorados o corregidos 
por las administraciones locales, tales como equipamientos públicos, como se presenta en un ejemplo más adelante).

En este ámbito cabe incluir, pues, no solamente las iniciativas de generación de visores locales conteniendo información de interés, como por ejemplo localizando los eventos municipales, creando las capas con rutas turísticas, etc., sino también aquellas otras actividades que no se reflejan directamente en un producto visual, pero que permiten mejorar la gestión administrativa a partir de los supuestos y principios de la colaboración, como se ilustrará más adelante con el ejemplo de mantenimiento y mejora de la base de equipamientos públicos y actualización del mapa callejero.

Las actividades de VGI se han visto posibilitadas gracias a la existencia de unos recursos tecnológicos al alcance de usuarios finales, gratuitos y de fácil manipulación ${ }^{1}$. Así la aportación de información por parte de usuarios no especializados, mediante plataformas y sistemas en el contexto de Internet, y su compartición con otros usuarios bien directamente bien a través de organizaciones intermediarias, se materializa y se consolida. No siendo ajeno al fenómeno el desarrollo del paradigma de las Infraestructuras de Datos Espaciales que organizan, formalizan, estandarizan e impulsan el uso de la Geoinformación con una visión de la información y los servicios distribuidos en la red y compartidos por todos.

Para fomentar este enfoque VGI, el Centro de Soporte de la IDEC, en colaboración con el Consorcio de Administración Abierta (E-Gov de Cataluña), puso a disposición de los usuarios locales y de entes públicos unos recursos fáciles de usar. En este escenario, la simplicidad es el factor más importante: no debería haber ninguna necesidad de ninguna habilidad especial para añadir o editar nuevos datos sobre las capas de referencia cartográficas. Siguiendo este principio, la $\mathrm{PRG}^{2}$ —Plataforma de Recursos de Geoinformación- de la IDEC ha ido reuniendo unos pocos recursos simples y útiles para el uso de las entidades locales. Esto incluye funcionalidades tales como la personalización de visualizadores, la creación de mapas temáticos, la edición de objetos geográficos (puntos, líneas, polígonos), etc.

En resumen, la «Plataforma de Recursos de Geoinformación» (PRG), permite el acceso a capas de información de diversos productores institucionales y ofrece un conjunto de herramientas y aplicaciones desarrolladas expresamente para su explotación por parte de las entidades locales en el marco de la

\footnotetext{
${ }^{1}$ http://www.ala.org/magirt/sites/ala.org.magirt/files/content/publicationsab/VGI\%20 MAGIRT\%20EP\%2012-postfinal.pdf

${ }^{2}$ Demo de la aplicación/Aplicación: http://www.geolocal.cat/PRG/eines.action
} 
mencionada IDE local. La PRG contribuye a ampliar el conocimiento y la utilización de los recursos IDE, y para mejorar la eficiencia en la gestión de los activos públicos debido a la reutilización de la información y las aplicaciones.

Algunos ejemplos ayudarán a explicar el estado de la situación.

\section{II.1. Ejemplo 1: Información ciudadana}

En los últimos años, varios municipios catalanes, utilizando la PRG, han creado y publicado nuevas colecciones de datos relacionados con su territorio.

Figura 1. Visualizador generado y personalizado con la $P R G$

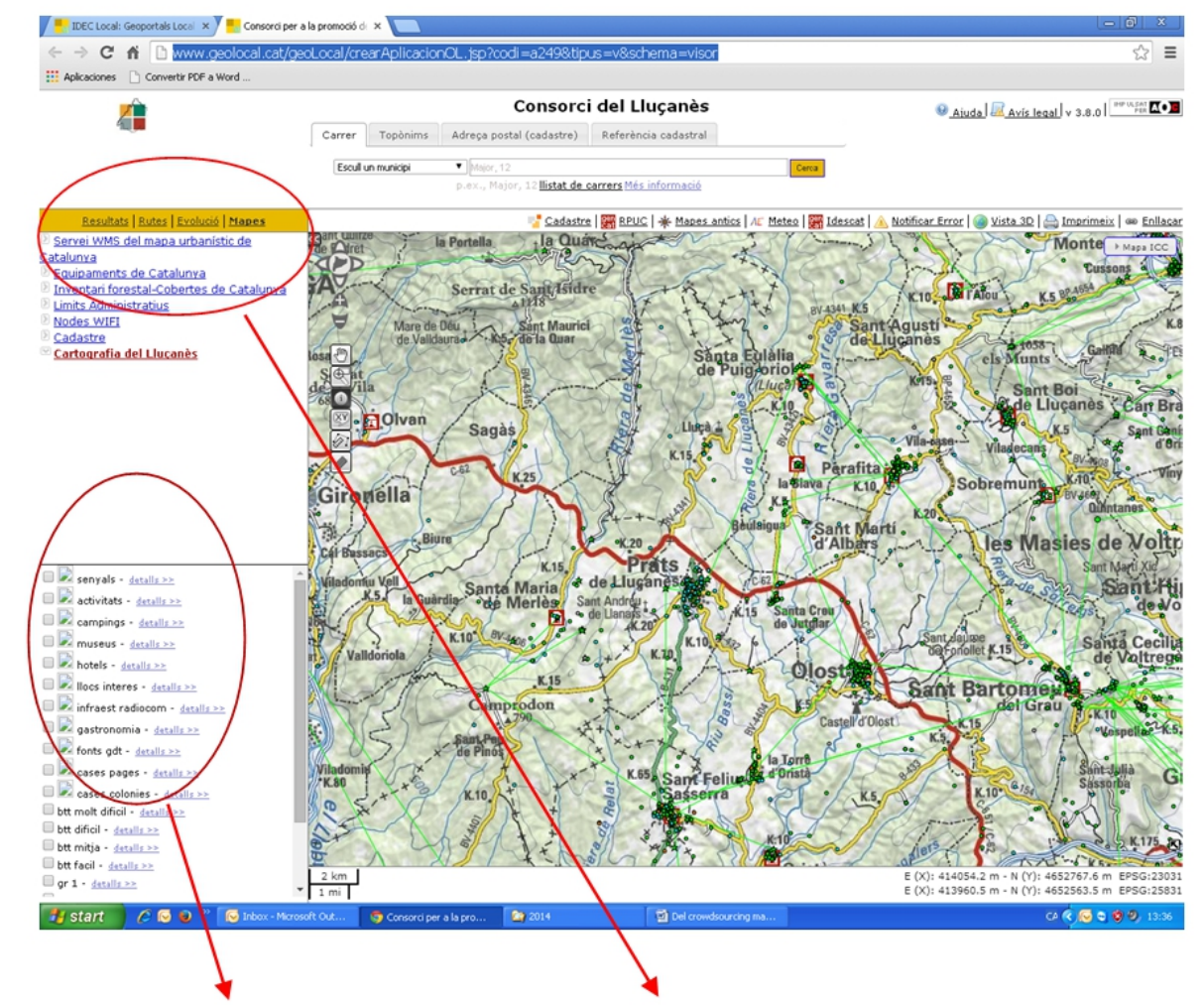

Capas creadas por el Ente local: rutas bus y stops, rutas turísticas, mercados, comunicaciones, casas de payés, etc.
Capas aportadas por los recursos de la red IDEC: Urbanismos, equipamientos, catastro, nodos Wifi, inventario forestal...

FUENTE: Plataforma de Recursos de Geoinformación 
Las capas de información se han publicado como servicios web WMS en Internet. La nueva información no se relaciona, en principio, con las responsabilidades administrativas formales del municipio, sino que obedece a la voluntad de facilitar más detallada información y servicios al ciudadano, tales como paradas de autobuses, circuitos turísticos, el patrimonio cultural, zonas protegidas, etc.

En la mayoría de los casos, la creación y publicación del tipo de información antes mencionado no responde a ningún plan municipal formal: sólo responde a la iniciativa de los «voluntarios» o funcionarios entusiastas debidamente autorizadas a tal efecto, que quieren mejorar la comunicación con el ciudadano y enriquecer su propio trabajo.

El siguiente ejemplo pertenece a un Ente Local, un consorcio de varios municipios: en su página web han publicado un geoportal que da acceso a información de base, procedente de WMS accesibles a través de la IDEC, así como diversas capas temáticas con datos de interés ciudadano, generadas por el referido Consorcio, mediante las ya explicitadas herramientas PRG.

\section{II.2. Ejemplo 2: Colaboración en el mantenimiento y mejora de la Base de Equipamientos Públicos}

Desde julio de 2008, unos 80 municipios catalanes han validado y mejorado las bases de datos de equipamientos (hospitales, oficinas públicas, escuelas, ...). Dicha información es facilitada por diversos Departamentos de la Generalitat, integrándose en una BdDatos de equipamientos (también accesible como «open data») y convertida por el CS IDEC en una capa mapificada después de un proceso de geocodificación de los datos, capas que son puestas a disposición de los entes locales juntamente con las aplicaciones para su edición.

Usando la PRG acceden a una aplicación basada en el estándar OGC WFS$\mathrm{T}$ desarrollado por IDEC. Como en el caso anterior, su aportación es totalmente voluntaria. La operativa puede consistir en añadir nuevos puntos correspondientes a otros equipamientos de gestión local, en la modificación de su localización (tema más frecuente) o bien en la corrección de algunos atributos. La actualización de la base de datos anteriormente mencionada se consigue ciertamente, de forma económica, muy cómoda y fácil. Además, se garantiza la validez de la información. Esta experiencia puede ser considerada como el inicio de la integración de ciertas actividades basadas en VGI «administrativa» en los procesos SIG formales o procedimientos administrativos. Actualmente se están diseñando los procedimientos para que las referidas modificaciones puedan revertir en los correspondientes Departamentos competentes, cerrando así el ciclo. 
Figura 2. Visualizador con la edición de la Base de Equipamientos Públicos

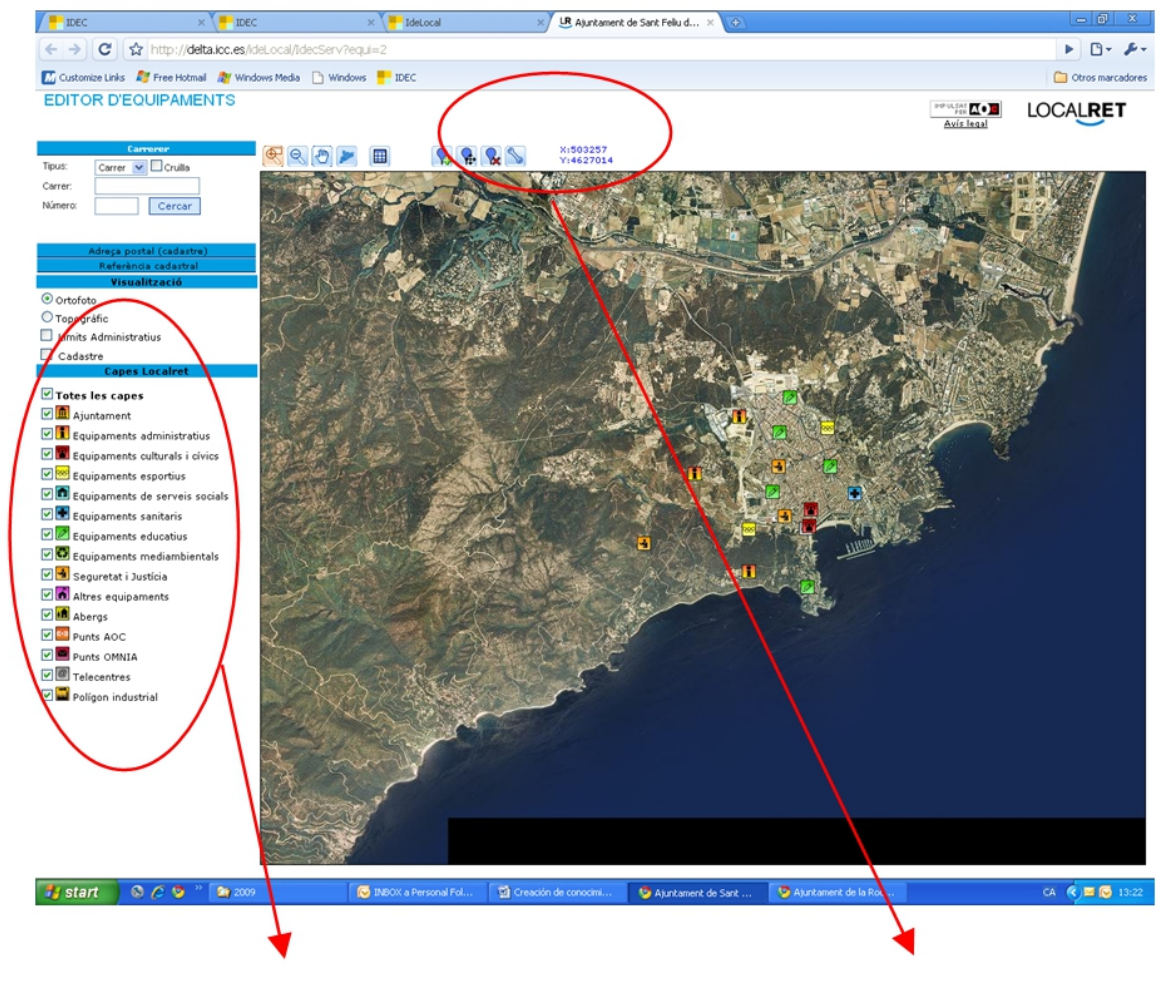

Herramientas para la edición de equi-

Equipamientos pamientos

FUENTE: Plataforma de Recursos de Geoinformación

Las herramientas de edición permiten tanto crear nuevos puntos de localización de equipamientos, desplegándose un formulario para la entrada de los atributos o características del equipamiento generado, como eliminar puntos, así como modificar datos de los mismos, tanto referentes a su localización (el punto de localización puede ser desplazado mediante el cursor) como a cualquiera de sus atributos.

\section{II.3. Ejemplo 3: Cooperación entre administraciones mediante nue- vos flujos de trabajo basados en herramientas VGI. Actualización del callejero.}

Los municipios tienen exclusiva competencia sobre la denominación de las calles de su término, por lo que disponen de la información más actualizada, siendo la mejor fuente para la recopilación de información para crear y actualizar la base de datos del callejero. Desafortunadamente, en muy pocos casos 
los datos están disponibles en una forma tal que puede ser fácilmente cargados en bases de datos. Habitualmente la información es enviada por el ayuntamiento a los organismos que la precisan, mediante soporte papel y en formato de listados y texto.

El Instituto Cartográfico y Geológico de Catalunya (IGCG) es la entidad responsable del Gobierno autónomo para la generación y publicación del callejero municipal del territorio de Catalunya. Para ello recibe información al respecto de diversos municipios que gestionan Sistemas de Información propios, así como notificaciones escritas (o verbales, en ocasiones) de otros municipios sin capacidades de gestión propio en este ámbito. Así pues, por una parte se intenta integrar la información digital procedente de los primeros, mediante transformación de formatos y otras operaciones hasta su incorporación en la BdDatos de calles, y por otra parte con medios propios o ajenos se realiza la edición de los mapas callejeros del segundo tipo de municipios.

Con el fin de mejorar estos flujos de trabajo para el proceso de actualización y aumentar la colaboración entre los municipios y el ICGC, nuevos flujos de trabajo se han diseñando para los escenarios descritos anteriormente. En particular, referente a la actualización de los mapas callejeros de los pequeños y medianos municipios que no disponen de sistemas digitales para ello, se ha desarrollado una aplicación integrada en la PRG que permite a los técnicos municipales, de una manera muy intuitiva y simple, dibujar sobre la cartografía 1:5.000 (o, eventualmente, 1:1000) digital los puntos o las líneas que se corresponden con los portales o los ejes de calle que son objeto de edición o modificación, complementándose con un formulario donde se explicitan las operaciones y los atributos. Dicha operación, una vez finalizada, genera una modificación a la BdD de «Observación» de calles, de manera muy similar a la que se ha expuesto antes en la descripción de la actualización de los equipamientos, la cual, una vez validada, comprobada y ejecutada por los técnicos del ICGC será incorporada en la BdD oficial. Ahorrándose así un tiempo ciertamente importante de gestión documental (envío de croquis, o de planos, con las modificaciones, comunicaciones, etc.) del proceso, y facilitando la operativa posterior que lleva a cabo el ICGC.

Un caso similar se está actualmente gestionando para la actualización de los caminos rurales.

En resumen, hemos descrito tres escenarios simples similares a muchos otros que se llevan a cabo bajo el paradigma del trabajo voluntario. No hay duda de que las posibilidades y las repercusiones de la creación del conocimiento geográfico en Internet son más que impresionantes. Pero además de 
ello, la arquitectura en red de una IDE puede dar soporte al trabajo colaborativo entre administraciones, acogiendo y materializando iniciativas que surgen inicialmente de problemas concretos que son abordados con alternativas tecnológicas nuevas, gracias a la predisposición de los profesionales responsables de dichos organismos, con cuyo concurso determinados proyectos, que podríamos enmarcar en el ámbito del voluntariado, pueden ser posibles.

\section{EL SIEMPRE PRESENTE PROBLEMA DE LA CALIDAD}

Uno de los problemas comunes de la VGI es la calidad (en términos de actualización, la resolución, la certeza...) asociada a estas contribuciones voluntarias de los ciudadanos, ya que es muy común que la información no pase ningún procedimiento de control de calidad, norma o supervisión. En el caso que nos ocupa, este problema es mucho menos preocupante, ya que se trata de información pública creada por entidades públicas o por los servidores públi$\cos$ de estas organizaciones que han sido debidamente autorizadas a tal efecto para el beneficio de información de los ciudadanos o la mejora de los procesos internos. También representa un incentivo para los políticos y funcionarios locales, ya que un importante valor agregado se obtiene a bajo costo.

Una ventaja importante del uso de la PRG como herramienta para la VGI es que se basa en la propia base de referencia cartográfica común, que es la cartografía «oficial», garantizando que los objetos creados «encajan» en el territorio, con independencia de quien sea la fuente de información temática. Esto no siempre es cierto en Globos Virtuales. Del mismo modo, la información generada permanece almacenada en los servidores que soportan la PRG (a diferencia de lo que ocurre en Globos Virtuales, en que los datos son almacenados en una «nube» no accesible directamente fuera del entorno de aplicación del G.V.) lo que permite la extracción de datos y otras posibilidades de análisis y explotación, al ser accesibles vía geoservicios estándar WMS-WFS.

\section{CONCLUSIONES}

Constatamos la existencia de una incipiente actividad de cooperación voluntaria de y entre muchos tipos diferentes de organizaciones, que no sólo es consecuencia de decisiones formales de la jerarquía superior de la organización, sino que, en muchos casos, surge a partir de los niveles técnicos de las mismas. Las aportaciones «no oficiales», tal como se ha expuesto, pueden tener una gran repercusión en la forma de abordar las relaciones inter-administraciones en un mundo digital.

El voluntariado institucional en IG se da en Entidades públicas que, con el impulso de quienes trabajan en las mismas, participan generando, mejorando 
y publicando información de interés público, aunque fuera del ámbito competencial de su gestión. Lo que contribuye a allanar el camino a nuevos y más formales procesos de cooperación entre los diferentes niveles de las administraciones públicas como las entidades locales y los gobiernos regionales.

Estamos seguros de que al considerar las IDEs como una plataforma de recursos a los que los usuarios pueden contribuir con nueva información tendrá un impacto social y económico indudable en un futuro más o menos inmediato.

Avanzamos (esperemos) hacia un modelo de administración teóricamente más liviano, más eficaz y eficiente, mediante la adopción informal de instrumentos de productividad (en general independientes de los SI internos corporativos) al alcance de las personas, $y$, en el caso que planteamos, de los funcionarios de entidades públicas. Así que la propuesta de nuevas ofertas en el ámbito de la cooperación inter-administraciones es, en la actualidad, muy posible gracias a las tecnologías interoperables y la disponibilidad de datos geográficos y geoservicios, junto con las referidas herramientas, y está generando ya resultados concretos, cuantificables y sostenidos.

\section{BIBLIOGRAFÍA Y FUENTES}

Coleman, D. J.; Georgiadou, Y. y Labonte, J. (2009): «Volunteered Geographic Information: The Nature and Motivation of Produsers». International Journal of Spatial Data Infrastructures Research, 2009, Vol. 4, 332-358.

http://ijsdir.jrc.ec.europa.eu/index.php/ijsdir/article/viewFile/140/223

Craglia, M. y Campagna, M. (Editores) (2009): Advanced Regional Spatial Data Infrastructures in Europe. Joint Research Centre. Institute for Environment and Sustainability. Ispra.

http://inspire.ec.europa.eu/documents/Study_and_Workshop_Reports/arsdi_repo rt.pdf

DieTZ, C. y SuH, J. (2012): Volunteered Geographic Information. Selected Web Resources. MAGIRT Electronic Publication Series No. 12.2012.

http://www.ala.org/magirt/sites/ala.org.magirt/files/content/publicationsab/VGI\%20MAGIRT\%20EP\%2012-postfinal.pdf

Garcia Almirall, P.; Moix Bergadè, M.; Queraltó Ros, P. y Craglia, M. (Editor) (2008): The Socio-Economic Impact of the Spatial Data Infrastructure of Catalonia report. Joint Research Centre. Institute for Environment and Sustainability. Ispra.

http://inspire.ec.europa.eu/reports/Study_reports/catalonia impact_study report.pdf

GOODCHILD, M.F. (2008): «Commentary: whither VGI?» GeoJournal 72, 239-244. [458].

http://www.geog.ucsb.edu/ good/papers/458.pdf 
SuI, D.Z.; Elwood, S. y GoodCHILD, M.F. (edit.) (2012): Crowdsourcing Geographic Knowledge: Volunteered Geographic Information (VGI) in Theory and Practice. New York: Springer. [534]

JACKSON, M.J.; RAHEMTULla, H.A.; MORLEY, J. (2010): «The Synergistic Use of Authenticated and Crowd-Sourced Data for Emergency Response». International Workshop on Validation of Geo-Information Products for Crisis Management. VALgEO 2010. JRC, Ispra, 11-13 October 2010; 91-99.

http://ipsc.jrc.ec.europa.eu/fileadmin/repository/globesec/isferea/docs/Proceedings of VALgEO2010.pdf

Wikipedia: Información Geográfica Voluntaria - Wikipedia, la enciclopedia libre. 